\title{
ESTUDO DA TOXICIDADE DE EFLUENTE TÊXTIL TRATADO POR FOTO-FENTON ARTIFICIAL UTILIZANDO AS ESPÉCIES LACTUCA SATIVA E ARTEMIA SALINA
}

\author{
Soraya Moreno Palácio \\ Daniele Alves Nogueira ${ }^{2}$ \\ Diego Ricieri Manenti ${ }^{3}$ \\ Aparecido Nivaldo Módenes ${ }^{1}$ \\ Fernando Rodolfo Espinoza Quiñones ${ }^{l}$ \\ Fernando Henrique Borba
}

\begin{abstract}
Resumo: Este trabalho apresenta a análise da toxicidade de efluente têxtil tratado por processo fotoFenton com irradiação artificial, utilizando alface (Lactuca sativa) e micro-crustáceos (Artemia salina) como organismos-teste. Foram retiradas alíquotas do efluente nos tempos de tratamento de 0, 5, 10, 15, 45, 60 e 90 minutos para análise da toxicidade, demanda química de oxigênio (DQO), cor e turbidez. Durante o experimento de toxicidade determinou-se a inibição do crescimento das raízes e radículas da alface, o efeito sobre a germinação das sementes e o efeito sobre a mortalidade dos micro-crustáceos, além da concentração letal $\left(\mathrm{DL}_{50}\right)$ para cada espécie. Analisando os resultados obtidos, verificou-se que o tratamento foto-Fenton artificial não reduziu a toxicidade do efluente têxtil; entretanto, na DQO, cor e turbidez, obtiveram-se reduçôes acima de $90 \%$ nos primeiros 45 minutos de irradiação.
\end{abstract}

Palavras-chave: Bioensaio, Efluente Têxtil, Foto-Fenton.

Abstract: In this paper, the toxicity of textile wastewater, treated by photo-Fenton process with artificial irradiation using lettuce (Lactuca sativa) and brine shrimp (Artemia Salina) as test organisms was analyzed. Effluent aliquots were taken at the treatment times of 0, 5, 10, 15, 45, 60 and 90 minutes for toxicity, chemical oxygen demand (COD), color and turbidity analysis. During the toxicity experiment were determined the inhibition of growth of lettuce roots and rootlets, the effect on seed germination and the effect on mortality of brine shrimp, beyond the lethal concentration $\left(\mathrm{LD}_{50}\right)$ for each species. Analyzing the results, was observed that photo-Fenton treatment with artificial UV irradiation did not reduced the textile effluent toxicity; however, COD, color and turbidity reductions above $90 \%$ were observed in the first $45 \mathrm{~min}$ of irradiation.

Keywords: Bioassay, Textile Effluent, Photo-Fenton.

\footnotetext{
Programa de Pós-Graduação em Engenharia Química da Universidade Estadual do Oeste do Paraná.

Curso de Graduação em Engenharia Química da Universidade Estadual do Oeste do Paraná.

3 Programa de Pós-Graduação em Engenharia Química da Universidade Estadual de Maringá.
} 


\section{INTRODUÇÃO}

O setor têxtil é um dos segmentos industriais mais poluidores de águas superficiais e seus processos são responsáveis por consumirem elevadas quantidades de água que posteriormente são convertidas em efluentes. Além de corantes, pigmentos e produtos auxiliares, os efluentes têxteis possuem grandes quantidades de compostos orgânicos e inorgânicos, que quando associados, apresentam toxicidade à vida aquática, diminuindo o oxigênio dissolvido e modificando as propriedades e características físicas dos cursos d'água (Braile \& Cavalcante 1993). Nestas circunstâncias, torna-se necessária a aplicação de métodos eficientes para a degradação dos efluentes têxteis, como por exemplo, os métodos oxidativos avançados (Torrades et al. 2004).

Os métodos oxidativos avançados incluem processos como $\mathrm{UV} / \mathrm{H}_{2} \mathrm{O}_{2}, \mathrm{TiO}_{2} / \mathrm{UV}$, Eletro-Fenton, Fenton e foto-Fenton (Vandevivere et al. 1998; Sauer et al. 2006; Schrank et al. 2004; Gozzo 2005 e Borba 2010). Estes processos são, em geral, baseados na formação do radical $\mathrm{OH}$ que atua oxidando os poluentes orgânicos a $\mathrm{CO}_{2}$, $\mathrm{H}_{2} \mathrm{O}$ e íons inorgânicos.

Nogueira et al. (2007) descreveram a sequência de reaçôes que ocorrem no processo foto-Fenton com irradiação artificial (FFA), que se inicia com a formação de aquo-complexos de $\mathrm{Fe}(\mathrm{III})$, conforme reação 1 . Nestes aquo-complexos, ocorre a redução do $\mathrm{Fe}(\mathrm{III})$ à $\mathrm{Fe}(\mathrm{II})$ e a oxidação do ligante com formação do radical $\mathrm{OH}$ pela ação da radiação UV/visível (reação 2).

$$
\begin{gathered}
\mathrm{Fe}^{3+}+\mathrm{H}_{2} \mathrm{O} \rightarrow \mathrm{Fe}(\mathrm{OH})^{2+}+\mathrm{H}^{+} \\
\mathrm{Fe}(\mathrm{OH})^{2+}+\mathrm{hv}(\text { vis }) \rightarrow \mathrm{Fe}^{2+}+{ }^{\cdot} \mathrm{OH}
\end{gathered}
$$

$\mathrm{Na}$ presença de peróxido de hidrogênio o Fe(II) gerado durante a irradiação, é oxidado a $\mathrm{Fe}(\mathrm{III})$ produzindo mais um radical $\mathrm{OH}$ (reação 3).

$$
\mathrm{Fe}^{2+}+2 \mathrm{H}_{2} \mathrm{O}_{2} \rightarrow \mathrm{Fe}^{3+}+\cdot \mathrm{OH}+\mathrm{OH}^{-}
$$

Os sistemas de tratamento de efluentes são monitorados convencionalmente por medidas indiretas, não específicas, de carbono orgânico, como a Demanda Bioquímica de Oxigênio (DBO), Demanda Química de Oxigênio (DQO) e Oxigênio Dissolvido (OD). Entretanto, estas análises físico-químicas não são capazes de distinguir entre as substâncias que afetam os sistemas biológicos e as que são inertes no ambiente e, desta forma, não são suficientes para avaliar o potencial de risco ambiental dos contaminantes (Ronco et al. 2004).

Segundo Kapanen \& Itavaara (2001), os testes de toxicidade podem ser classificados de acordo com o tempo de exposição (agudo ou crônico), o modo do efeito (morte, crescimento ou reprodução) ou a resposta do efeito (letal ou sub-letal).

$\mathrm{O}$ indicador de letalidade mais comummente utilizado é o $\underline{\mathrm{DL}}_{50}$, correspondente à dose capaz de matar 50\% dos indivíduos de uma população em teste. Segundo a CETESB (1990), essa é a resposta considerada mais significativa para ser extrapolada a uma população. Os efeitos que continuam por longo tempo se traduzem como efeitos crônicos ou sub-letais e podem abranger parte ou todo o ciclo de vida dos organismos.

Plantas sensíveis à substâncias tóxicas podem ser utilizadas como indicadores da qualidade do meio, constituindo-se bioensaios de fitotoxicidade. O National Water Research Institute, localizado no Canadá (Dutka 1989), recomenda o emprego de sementes de alface em testes de toxicidade de efluentes, solos ou sedimentos, devido ao crescimento rápido e a pouca reserva de energia necessária para sua germinação. Comumente avalia-se a inibição do processo germinativo e o alongamento das raízes, que ocorre na presença de pequenas concentrações do composto tóxico, tornando este organismo um indicador mais sensível aos efeitos biológicos.

A Artemia salina, um micro-crustáceo de água salgada, destaca-se por compor um bioensaio de toxicidade de baixo custo, rapidez na obtenção dos resultados e por não exigir técnicas assépticas. Em função de seu habitat natural, representa um importante indicador biológico para meios com elevadas concentrações de sais (Carvalho et al. 1988).

Palácio et al. (2010) realizaram análises de toxicidade em efluente têxtil tratado por eletrocoagulação, utilizando como organismos-teste as espécies Lactuca sativa e Artemia salina. Neste trabalho, foi evidenciado que o tratamento do efluente reduziu de forma satisfatória a toxicidade sobre estes organismos.

O presente trabalho teve como objetivo avaliar a toxicidade de um efluente têxtil tratado 
pelo processo foto-Fenton artificial, utilizando as espécies Lactuca sativa e Artemia salina como organismos-teste, comparando os resultados obtidos com a redução da DQO, cor e turbidez.

\section{METODOLOGIA}

O efluente têxtil (ET) foi submetido ao tratamento pelo processo foto-Fenton artificial (FFA) em reator de escala laboratorial utilizando o sistema batelada. $\mathrm{O}$ reator consistiu em um béquer de borossilicato de $200 \mathrm{~mL}(9,3 \mathrm{~cm}$ de altura x 7,8 cm diâmetro) e um agitador magnético (ARE UNI-3650) para homogeneização das amostras. A irradiação artificial do ET foi realizada em uma câmara contendo três reatores. A Figura 1 apresenta o esquema da câmara utilizada na reação foto-Fenton.

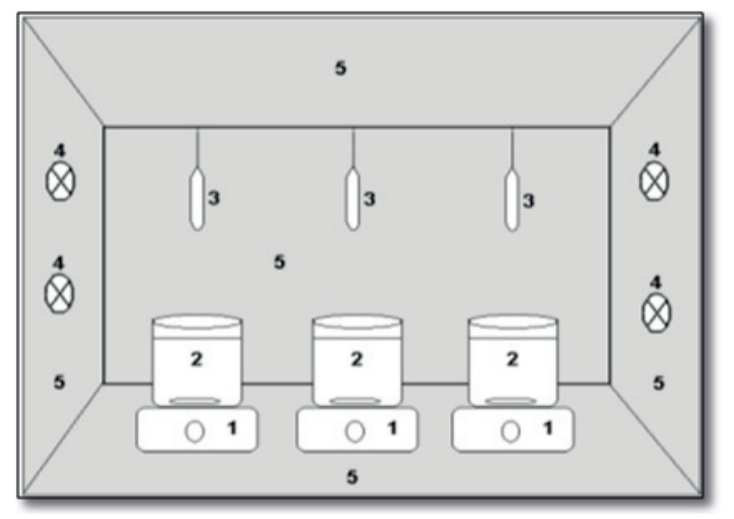

Figura 1. Sistema utilizado para as reaçôes fotoFenton com irradiação artificial. Sendo: 1 = agitadores magnéticos; 2 = reatores foto-Fenton; 3 = lâmpadas de mercúrio; 4 = ventiladores; $5=$ revestimento em papel alumínio.

Manenti (2011) determinou as condições ótimas experimentais para o tratamento do efluente têxtil pelo processo foto-Fenton com irradiação artificial. O ET foi submetido à irradiação utilizando lâmpadas de vapor de mercúrio de $250 \mathrm{~W}$. As condições operacionais determinadas foram: $\left[\mathrm{Fe}^{2+}\right]=0,05 \mathrm{~g} \mathrm{~L}^{-1} ;\left[\mathrm{H}_{2} \mathrm{O}_{2}\right]=6 \mathrm{~g} \mathrm{~L}^{-1}$ e $\mathrm{pH}$ inicial 3,0.

O ET foi irradiado por 90 minutos, sendo retiradas amostras nos tempos de irradiação 5, 10, 15, 45, 60 e 90 minutos. As amostras irradiadas e o efluente bruto foram filtrados utilizando-se membrana de acetato de celulose com diâmetro de poro de 0,45 $\mu \mathrm{m}$ e submetidas às análises de DQO, cor e turbidez segundo metodologia descrita em
APHA (1998). As concentrações de peróxido de hidrogênio residuais foram analisadas segundo a metodologia descrita em Silva et al. 2004.

Para a realização dos testes de toxicidade, as amostras foram armazenadas por aproximadamente 30 dias, em recipientes escuros e sob refrigeração $\left(\sim 10^{\circ} \mathrm{C}\right)$. Após este período, ajustou-se o $\mathrm{pH}$ das amostras para 7,0.

\subsection{BIOENSAIOS COM LACTUCA SATIVA}

Os testes de fitotoxicidade foram conduzidos segundo a metodologia descrita em Sobrero \& Ronco (2004). Utilizaram-se sementes de Lactuca sativa Aurélia, com percentual de germinação de $98 \%$ (fornecido pelo fabricante).

As amostras (efluente bruto e os tratados) sofreram diluições utilizando-se água dura reconstituída (APHA 1998). Os percentuais de efluente em cada diluição foram 1, 3, 10, 30 e 100\%. Água dura foi utilizada como controle negativo.

Placas de Petry de $9 \mathrm{~cm}$ de diâmetro foram preparadas com papel de filtro qualitativo de diâmetro apropriado, onde foram depositadas espaçadamente vinte sementes de alface e umedecidas com as diluiçôes preparadas anteriormente, em triplicata.

As placas foram acondicionadas em sacos plásticos para evitar a perda de umidade e levadas à incubadora com temperatura de $22^{\circ} \mathrm{C} \pm 2$, no escuro, por um período de 120 h. Após a incubação contou-se o número de sementes germinadas e mediram-se os comprimentos das raízes e radículas.

$O$ percentual de germinação relativa para cada diluição foi calculado através da equação 1 .

$$
\% \mathrm{GR}=\frac{\mathrm{N}^{\circ} \mathrm{SGA}}{\mathrm{N}^{\circ} \mathrm{SGC}} * 100
$$

Os percentuais de inibição do crescimento relativo das raízes (\% ICRRz) e das radículas (\% ICRRd), foram calculados a partir dos valores médios para cada diluição, utilizando-se as equações 2 e 3.

$$
\begin{aligned}
& \text { \%ICRRz }=\frac{\text { MCRzC }- \text { MCRzA }}{\text { MCRzC }} * 100 \\
& \% \text { ICRRd }=\frac{\text { MCRdC }- \text { MCRdA }}{\text { MCRdC }} * 100
\end{aligned}
$$


Os valores de $\mathrm{DL}_{50}$, para cada amostra, foram estimados conforme a metodologia descrita por Hamilton et al. (1997), utilizando-se o software Trimmed Spearman-Karber Method Versão 1.5.

\subsection{BIOENSAIOS COM ARTEMIA SALINA}

Os cistos de Artemia salina foram eclodidos em uma solução nutritiva descrita por Meyer et al. (1982), contendo 23,0 $\pm 0,1 \mathrm{~g}$ de $\mathrm{NaCl} ; 11,0$ $\pm 0,1 \mathrm{~g}$ de $\mathrm{MgCl}_{2} \cdot 6 \mathrm{H}_{2} \mathrm{O} ; 4,0 \pm 0,1 \mathrm{~g}$ de $\mathrm{Na}_{2} \mathrm{SO}_{4}$ $1,3 \pm 0,1 \mathrm{~g} \mathrm{de} \mathrm{CaCl}_{2} .2 \mathrm{H}_{2} \mathrm{O}$ e $0,7 \pm 0,1 \mathrm{~g}$ de $\mathrm{KCl}$ em $1 \mathrm{~L}$ de água destilada, tendo o valor de $\mathrm{pH}$ ajustado para 9,0, antes da inoculação dos cistos, utilizando-se uma solução de $\mathrm{Na}_{2} \mathrm{CO}_{3}$. O tempo de eclosão foi de $48 \mathrm{~h}$.

O efluente bruto e os tratados foram diluídos utilizando-se 20, 40, 60, 80 e 100\% de efluente, em triplicata. A solução de Meyer foi utilizada como água de diluição e como controle negativo. Foram transferidos $5 \mathrm{~mL}$ de cada diluição para tubos de ensaio e adicionadas 10 larvas de Artemia salina em cada tubo. Os tubos foram incubados à temperatura ambiente na presença de luz por 24 h. Após este período, contou-se o número de organismos vivos em cada tubo e estimou-se a $\mathrm{DL}_{50}$ conforme descrito anteriormente.

\section{RESULTADOS E DISCUSSŌES}

Os resultados de toxicidade obtidos para a espécie Artemia salina mostraram que esta não foi capaz de se estabelecer no meio. Após 24 horas de incubação, foi constatado que não havia organismos sobreviventes em qualquer um dos tubos, independente da diluição ou tempo de irradiação das amostras.

Já a espécie Lactuca sativa mostrou-se capaz de se estabelecer em meio potencialmente tóxico e a se desenvolver parcialmente, apresentando efeitos letais (inibição da germinação) e sub-letais (inibição do desenvolvimento das raízes e das radículas).

Os resultados de germinação relativa para as sementes de alface são apresentados na Figura 2, onde podem ser observados os efeitos do tempo de irradiação e da diluição da amostra.

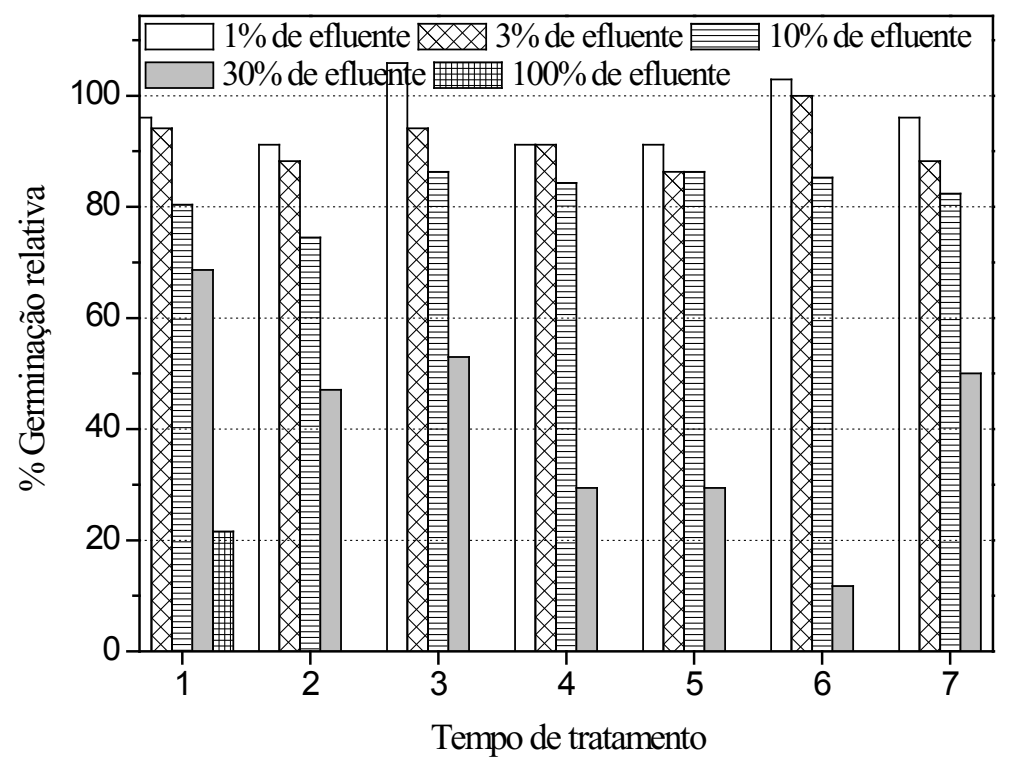

Figura 2. Germinação relativa ao controle negativo em função do tempo de irradiação do ET, onde (1) efluente bruto; (2) 5 min.; (3) 10 min.; (4) 15 min.; (5) 45 min.; (6) $60 \mathrm{~min} . \mathrm{e}(7) 90 \mathrm{~min}$. 
O percentual de germinação foi maior nas amostras mais diluídas, uma vez que a quantidade de substâncias tóxicas diminui com o aumento da diluição. Nas amostras que continham 100\% de efluente não houve germinação, independentemente do tempo de irradiação, exceto na amostra de efluente bruto, onde o percentual de germinação foi de $20 \%$, indicando que o tratamento do efluente têxtil pelo processo FFA produziu intermediários responsáveis pela elevação da toxicidade. Deste modo, as amostras sem diluição apresentaram elevados efeitos de letalidade.

Nas amostras onde houveram germinação foi possível calcular os percentuais de inibição do desenvolvimento das raízes e radículas das plântulas de alface. Os resultados obtidos são mostrados nas Figuras 3 e 4, respectivamente.

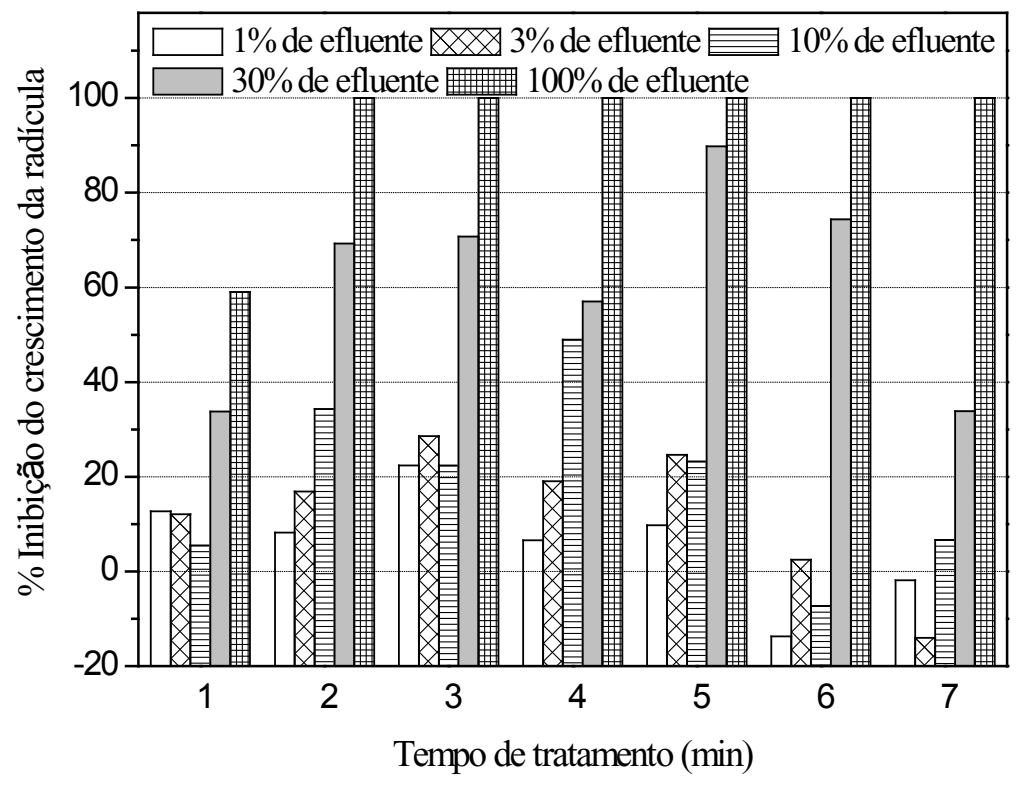

Figura 3. Inibição do crescimento da raiz relativo ao controle negativo, em função do tempo de irradiação do ET, onde (1) efluente bruto; (2) 5 min.; (3) 10 min.; (4) 15 min.; (5) 45 min.; (6) 60 min. e (7) 90 min.

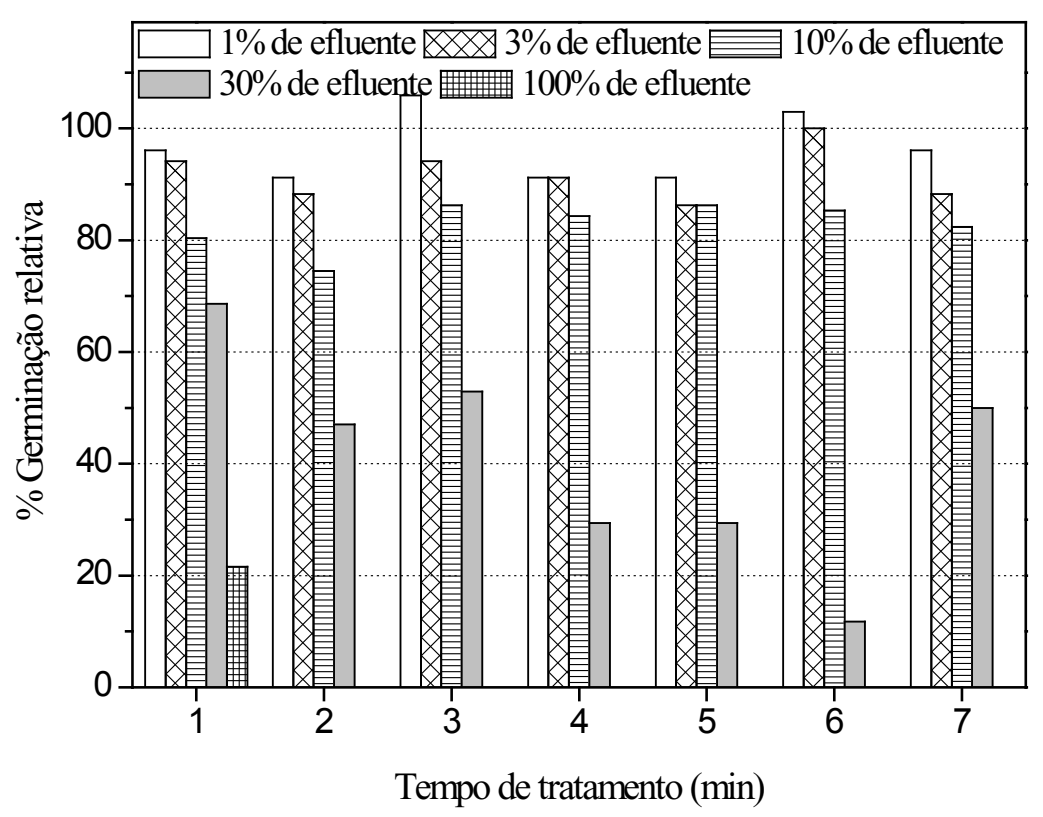

Figura 4. Inibição crescimento da radícula relativo ao controle negativo, em função do tempo de irradiação do ET, onde (1) efluente bruto; (2) 5 min.; (3) 10 min.; (4) 15 min.; (5) 45 min.; (6) 60 min. e (7) 90 min. 
As plântulas de alface sofreram menor inibição no crescimento das raízes e das radículas nas amostras mais diluídas, devido à menor concentração de substâncias tóxicas.

Ocorreram inibições negativas nas amostras retiradas aos 60 e 90 minutos de processo FFA (nas diluiçôes de 1, 3 e 10\%), decorrência do maior desenvolvimento das radículas nestas amostras do que no controle negativo.

A toxicidade do ET irradiado não pode ser atribuída à presença de peróxido de hidrogênio residual, uma vez que as análises de peróxido não acusaram a presença deste. $\mathrm{O}$ efeito sub-letal

Os valores de $\mathrm{DL}_{50}$ calculados para a espécie Lactuca sativa expostas ao ET são apresentados na Tabela 1. Não houve redução da letalidade dos organismos expostos ao ET ao longo do tratamento FFA. Após 90 minutos de irradiação observa-se uma alta toxicidade do ET tratado em relação ao ET bruto (tempo zero).

Tabela 1. Valores de $\mathrm{DL}_{50}$ para alíquotas de ET submetido ao processo FFA, utilizando a espécie Lactuca sativa.

\begin{tabular}{|c|c|c|}
\hline $\begin{array}{c}\text { Tempo de } \\
\text { irradiação } \\
\text { (min.) }\end{array}$ & $\mathrm{DL}_{50}(\%)$ & $\begin{array}{c}\text { Intervalo de } \\
\text { Confiança (95\%) }\end{array}$ \\
\hline 0 & 34 & $20-60$ \\
\hline 5 & 16 & $14-32$ \\
\hline 10 & 24 & $17-34$ \\
\hline 15 & 20 & $14-28$ \\
\hline 45 & 21 & $15-29$ \\
\hline 60 & 17 & $14-22$ \\
\hline 90 & 23 & $15-34$ \\
\hline
\end{tabular}

Os parâmetros físico-químicos DQO, cor e turbidez apresentaram reduções satisfatórias, conforme apresentado na Figura 5. Aos 45 minutos de irradiação, a DQO, cor e turbidez alcançaram reduçóes de 94, 99 e 97\% respectivamente em relação ao caracterizado para o ET bruto.

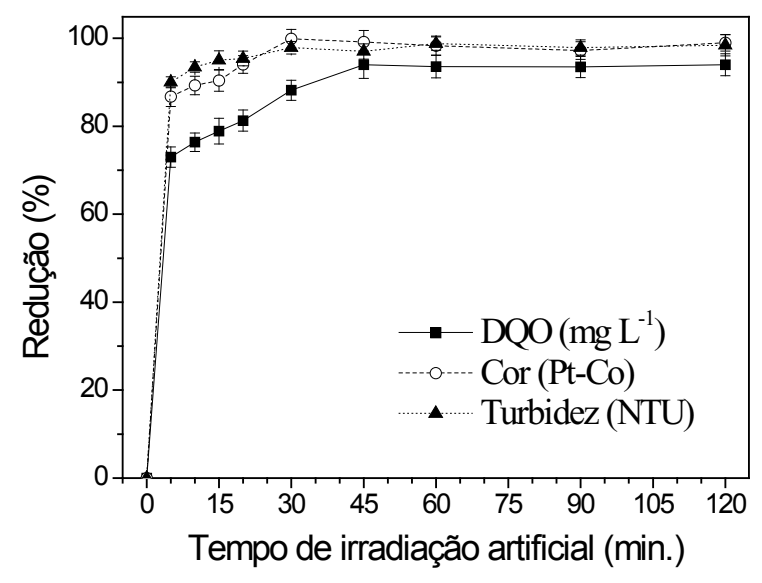

Figura 5. Reduções de DQO, cor e turbidez em função do tempo de irradiação do ET.

Os resultados de DQO, cor e turbidez quando comparados à $\mathrm{DL}_{50}$ mostraram que mesmo com uma redução significativa destes parâmetros, o ET não apresentou condições toxicológicas satisfatórias, apresentando-se altamente tóxico em todos os tempos de tratamento.

A elevada redução na DQO indica uma elevada redução da matéria orgânica degradável, porém a alta toxicidade sugere a presença de compostos tóxicos no efluente, que são refratários ao tratamento e não são detectados pela DQO.

\section{CONCLUSÕES}

O processo FFA aumentou a toxicidade do ET, contudo houve significativa redução nos valores de $\mathrm{DQO}$, cor e turbidez.

A utilização de diferentes organismos para avaliar a toxicidade do ET evidencia a sensibilidade particular de cada organismo num dado meio. No caso do ET tratado por FFA, ambas as espécies estudadas indicaram alta toxicidade do meio. Entretanto, a espécie Lactuca sativa apresentou menor sensibilidade, sendo possível determinar os valores de $\mathrm{DL}_{50}$ que variou entre 16 a $34 \%$, sendo maior no efluente bruto que nos tratados.

Os bioensaios realizados conjuntamente às análises físico-químicas fornecem informaçōes importantes para o monitoramento da qualidade do efluente a ser descartado, com o intuito de garantir a integridade do meio ambiente. 


\section{NOMENCLATURAS}

\begin{tabular}{|l|l|}
\hline GR & Germinação relativa \\
\hline ICRRd & $\begin{array}{l}\text { Inibição do crescimento relativo da } \\
\text { radícula }\end{array}$ \\
\hline ICRRz & $\begin{array}{l}\text { Inibição do crescimento relativo } \\
\text { da raiz }\end{array}$ \\
\hline MCRdA & $\begin{array}{l}\text { Média de crescimento das radículas } \\
\text { na amostra }\end{array}$ \\
\hline MCRdC & $\begin{array}{l}\text { Média de crescimento das radículas } \\
\text { no controle negativo }\end{array}$ \\
\hline MCRzA & $\begin{array}{l}\text { Média de crescimento das raízes na } \\
\text { amostra }\end{array}$ \\
\hline MCRzC & $\begin{array}{l}\text { Média de crescimento das raízes no } \\
\text { controle negativo }\end{array}$ \\
\hline SGA & $\begin{array}{l}\text { Número de sementes germinadas } \\
\text { na amostra }\end{array}$ \\
\hline SGC & $\begin{array}{l}\text { Número de sementes germinadas } \\
\text { no controle }\end{array}$ \\
\hline
\end{tabular}

\section{REFERÊNCIAS}

APHA: Standard methods for the examination of water and wastewater. 20th ed. Baltimore, MD: United Book Press, 1998.

Borba, F. H. Aplicação dos processos foto-Fenton e eletrofloculação no tratamento de efluente de curtume. Dissertação de Mestrado da Faculdade de Engenharia Química da Universidade Estadual do Oeste do Paraná, 2010.

Braile, P. M., Cavalcante, J. E. W. A. Manual de Tratamento de Águas Residuárias Industriais. 18. Ed. São Paulo: Companhia de Tecnologia de Saneamento Ambiental - CETESB, p. 764, 1993.

Carvalho, A. F. F. U., Melo, V. M. M., Aguiar, L. M. B. A., Matos, F. J. A. Avaliação da toxicidade de extratos de plantas medicinais através de bioensaio com Artemia salina. Leach. Cienc. Cult. v. 40, p.1109-1111, 1988.

COMPANHIA DE SANEAMENTO AMBIENTAL. Procedimentos para utilização de testes de toxicidade no controle de efluentes líquidos. São Paulo: CETESB, 1990.
Dutka, B. J. Methods microbiological for toxicological analysis of water, wastewater and sediments. National Water Research Institute, Burlington, Ontario, Canadá, p.127, 1989.

Gozzo, F. Radical and non-radical chemistry of Fenton like systems in presence of organic substances. Journal of Molecular Catalysis A: Chemical. v. 171, p. 1-22, 2005.

Hamilton, M. A., Russo, R.C., Thurston R.V. Trimmed Spearman Karber statistical method for estimating median lethal concentration in toxicity bioassays, Environ. Sci. Technol. v.11, p.714719, 1997.

Kapanen, A., Itavaara, M. Ecotoxicity tests for compost applications. Ecotoxicology and Environmental Safety, v. 49, p. 1-16, 2001.

Manenti, D. R. Tratamento de efluente têxtil sintético e real utilizando o processo foto-Fenton com irradiação artificial e solar. Dissertação de Mestrado da Faculdade de Engenharia Química da Universidade Estadual do Oeste do Paraná, 2011.

Meyer, B. N., Ferrigni, N. R., Putnam, J. E., Jacobsen, L. B., Nichols, D. E., Mclaughlin, J. L. Brine shrimp: a convenient general bioassay for active plant constituents, Planta Med. v.45, p. 3534, 1982.

Nogueira, R. F. P., Trovó, A. G., Silva, M. R. A., Villa, D. R. Fundamentos e aplicações ambientais dos processos fenton e foto-Fenton. Quim. Nova, v. 30, p 400-408, 2007.

Ronco, A., Báez, M. C. D., Granados, Y. P. In: Ensayos Toxicológicos y Métodos de Evaluación de Calidad de Aguas - Estandarización, Intercalibración, Resultados y Aplicaciones; Morales, G. C., ed.; Centro Internacional de Investigaciones para el Desarrollo: Ottawa, cap. 1, 2004.

Palácio, S. M., Espinoza-Quiñones, F. R., Módenes, A. N., Oliveira, C. C., Borba, F. H., Silva, F. G. Toxicity assessment from electro-coagulation treated-textile dye wastewaters by bioassays. Journal of Hazardous Materials, v. 172, p. 330-337, 2009. 
Sauer, T. P., Casaril, L., Oberziner, A. L. B., José, H. J., Moreira, R. F. P. M. Advanced oxidation process applied to tannery wastewater containing Direct Black 38 - Elimination and degradation Kinetics. Journal of Hazardous Materials, v. 135, p. 274-279, 2006.

Silva, M. R. A., Oliveira, M. C., Nogueira, R. F. P. Estudo da aplicação do processo Foto-Fenton solar na degradação de efluentes de industrias de tintas. Eclética Química, v. 29, p.19-26, 2004.

Sobrero, M. S. \& Ronco, A. Ensayo de toxicidad aguda con semillas de lechuga. In: Ensayos Toxicologicos y Métodos de Evaluacion de calidad de Aguas: Estandarización, intercalibración, resultados y aplicaciones. Faculdad de Ciencias Físicas e Matemáticas Ed. Chile, cap. 4, 2004.
Schrank, S. G., José, H. J., Moreira, R.F.P.M., Schroder, H. F. R. Elucidation of the behavior of tannery wastewater under advanced oxidation conditions. Chemosphere, v. 56, p. 411-423, 2004.

Torrades, F., Garcia-Montano, J., Garcia-Hortal, J. A., Domenech, X., Peral, J. Decolorization and mineralization of commercial reactive dyes under solar light assisted photo-Fenton conditions. Solar Energy, v.77, p.573-581, 2004.

Vandevivere, P. C., Bianchi, R., Verstraete, W. Treatment and reuse of wastewater from the textile wet-processing industry: review of emerging technologies. Journal of Chemical Technology Biotechnology, v.72, p. 289- 302, 1998. 